\title{
Size dependence of the bulk modulus of Si nanocrystals
}

\author{
B J ABDULLAH ${ }^{1, *}$, M S OMAR ${ }^{1}$ and Q JIANG ${ }^{2}$ \\ ${ }^{1}$ Department of Physics, College of Science, Salahaddin-Erbil University, Erbil, Kurdistan Region 31019, Iraq \\ ${ }^{2}$ Department of Material Science and Engineering, Jilin University, Changchun 130022, China \\ e-mail: botan_nano@yahoo.com
}

MS received 31 January 2016; revised 2 June 2018; accepted 1 August 2018; published online 12 September 2018

\begin{abstract}
This study investigates the effect of size on bulk modulus and its related parameters, including melting temperature and mass density based on the ratio number of surface atoms to that of its internal. The equation of bulk modulus in the bulk state $\mathrm{B}(\infty)$ is modified to include the related size-dependent parameters without any adjustable parameter, and is applied to $\mathrm{Si}$ nanocrystals. The bulk modulus $\mathrm{B}(\mathrm{r})$ decreases from $9.8 \times 10^{10} \mathrm{~N} \mathrm{~m}^{2}$ for the bulk state to $5.93 \times 10^{10} \mathrm{~N} \mathrm{~m}^{2}$ for a $5 \mathrm{~nm}$ diameter of Si nanoparticles. An inherent relation between bulk modulus and change of the lattice parameter in nanocrystals obtained from the variation in the surface to volume ratio, this leads to increase in the mean bond length. The effect of mass density and melting temperature on bulk modulus are also discussed. Calculated results for bulk modulus are verified by experimental as well as the available computer simulation data.
\end{abstract}

Keywords. Si; nanocrystals; bulk modulus; mass density; melting temperature.

\section{Introduction}

Size-dependent properties of nanocrystals (NCs) are one of the most important foundations of nanoscience and nanotechnology. NCs, including nanoparticles (NPs) nanowires (NWs) and nanofilms (NFs) are suitable and informative objects for investigating finite-size effects on various physical properties of solids [1-3]. In understanding the size-dependent property characterization at the nanoscale, theoretical considerations are important because of the partially accurate measurement techniques on these material properties. When the size of low-dimensional materials decreases to a nanometer size range, their thermodynamic, elastic, electronic, optic, magnetic, and catalytic properties are significantly altered when compared with that of bulk state and the property characterization of solids is no longer constant and often produces qualitatively new behavior. For example, the melting temperature $T_{m}(r)$ [4-7] and the Debye temperature $\theta_{D}(r)$ [8-10] decrease with size reduction of NCs.

The bulk modulus is an elastic property, which is the measure of resistance to volume change and is strongly related to both pressure and temperature for bulk materials $[11,12]$. The bulk modulus is important in measuring the hardness and provides fundamental basis to thermal and elastic properties of solids. In order to understand the thermodynamic properties and hence the elastic behavior of NCs more profoundly, it is very necessary to develop the nanoscale thermodynamic method. In this work, the

*For correspondence equation to calculate the bulk modulus $B(\infty)$ is modified for the calculations of nanoscale size dependence and is applied to Si for both NWs and NPs. The size effects on the bulk modulus are calculated and the results obtained are compared with the available computer simulation data. Furthermore, mass density also playing an important role in material growth, homogeneity and porosity [13-16] and its effect on bulk modulus is also discussed.

\section{Method of calculations}

In general, at room temperature and constant pressure, the bulk modulus $\mathrm{B}(\infty)$ is related to sound group velocity $v_{g}(\infty)$ and mass density $\rho(\infty)$ according to the equation:

$$
\mathrm{B}(\infty)=v_{g}^{2}(\infty) \rho(\infty)
$$

where $v_{g}(\infty)$ is the bulk group velocity and $\rho(\infty)$ is the bulk mass density in bulk state. Upon structure miniaturization to the nanometer regime, the bulk modulus $\mathrm{B}(\infty)$ similar to other parameters is no longer constant but changes with the shape and size of the solid $[17,18]$. Thus, $\mathrm{B}(\infty)$ is also a nanosize dependent and modifying the form to $\mathrm{B}(\mathrm{r})$, where $\mathrm{B}(\mathrm{r})$ for nanoscale state depends on the sound group velocity $v_{g}(r)$ and mass density $\rho(r)$, this yields the following form:

$$
\mathrm{B}(\mathrm{r})=v_{g}^{2}(r) \rho(r)
$$

In this equation, $v_{g}(r)$ is calculated according to the following relation [19]: 


$$
v_{g}(r)=v_{g}(\infty) \frac{\theta_{D}(\mathrm{r})}{\theta_{D}(\infty)}
$$

where $\theta(\infty)$ is the bulk Debye temperature and $\theta(r)$ is the size dependent Debye temperature. The $\theta_{D}(\mathrm{r})$ for $\mathrm{NCs}$ is calculated from the following relation $[6,9,19,20]$ :

$$
\theta_{D}(r)=\theta_{D}(\infty)\left[\frac{T_{m}(\mathrm{r})}{T_{m}(\infty)}\right]^{1 / 2}
$$

where $T_{m}(\infty)$ is the bulk melting point and $T_{m}(r)$ is the nanoscale size dependent melting point, which is calculated from the following relation [21]:

$$
\frac{T_{m}(r)}{T_{m}(\infty)}=\left(\frac{V(r)}{V(\infty)}\right)^{\frac{2}{3}} e^{\left(-\frac{2\left(S_{m}-R\right)}{3 R\left(\frac{r}{r_{c}}\right)-1}\right)}
$$

where $R$ is the ideal gas constant and is equal to (8.314 $\mathrm{J} \mathrm{K}^{\circ} \mathrm{mol}^{-1}$ ), $S_{m}$ is bulk overall melting entropy, $V(\infty)$ is the bulk lattice volume, which is equal to $a^{3}(\infty)$, and $V(r)$ is the nanoscale lattice volume, which is equal to $a^{3}(r)$. For nanoscale, the size-dependent lattice parameter $a(r)$ is given by [22]:

$$
a(r)=\frac{4}{\sqrt{3}} d_{\text {mean }}(r)
$$

where Eq. (6) is a general equation for cubic system and $d_{\text {mean }}(r)$ is the size-dependent mean bond length, which is calculated according to $d_{\text {mean }}(r)=h-\Delta d_{\text {mean }}(r)$, where $h$ is the atomic diameter of the crystal and $\Delta d_{\text {mean }}(r)$ is the increase in the mean bond length and is calculated as:

$$
\Delta \mathrm{d}_{\text {mean }}(\mathrm{r})=\Delta \mathrm{d}_{\text {mean }}\left(\mathrm{r}_{\mathrm{c}}\right)\left[\mathrm{e}^{\frac{-2\left(\mathrm{~S}_{m}-\mathrm{R}\right)}{3 R\left(\frac{\mathrm{r}}{\mathrm{r}_{\mathrm{o}}}-1\right)}}\right]^{\frac{1}{2}}
$$

Recalling $\Delta d_{\text {mean }}\left(r_{c}\right)$ as the maximum increase in the mean bond length and is equal to $(0.0988 \mathrm{~nm})$ for $\mathrm{Si}, \mathrm{r}_{\mathrm{o}}$ denotes a critical radius at which all atoms of the particle are located on the bulk surface and is calculated from $r_{o}=(3-d) h$. In this equation, $d=0$ for NPs where $r$ is the radius, $d=1$ for NWs where $r$ is the radius, and $d=2$ for NFs, where $r$ is the half thickness [23, 24]. The nanoscale size dependence for melting temperature $T_{m}(r)$ is shown in figure 1, which included volume ratio $V(r) / V(\infty)$. Figure 1 shows that the melting temperature decreases when the size is decreased for Si NPs.

For a low dimensional crystal system, geometric structure influences the properties of nanomaterial in which the excess volumes is a function of both dislocation density and grain size [25]. In 2016, Abdullah [26] reported the following model for calculating size-dependent mass density in NCs, which provides a direct dependence of mass density on NCs volume according to the following relation:

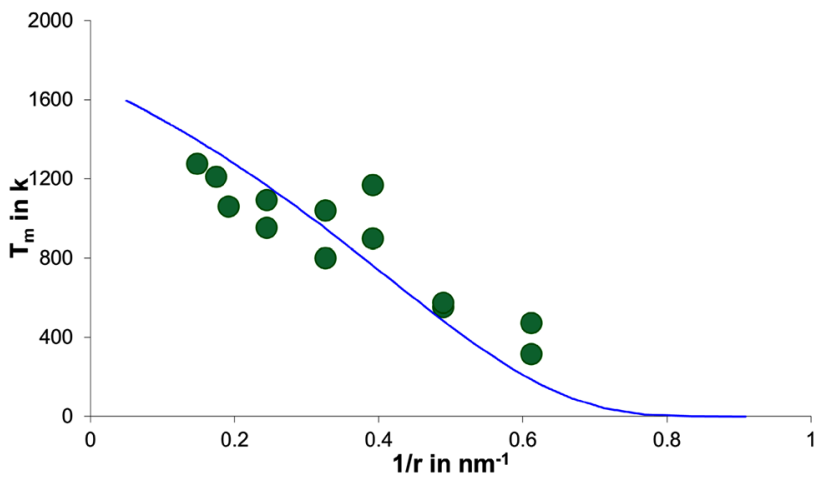

Figure 1. Melting temperature of Si NPs. The solid curve is obtained by Eq. (5). The symbols denote the experimental results [27].

Table 1. The relevant data used in the calculations for Si.

\begin{tabular}{lc}
\hline Parameters & $\mathrm{Si}$ \\
\hline$B(\infty) / \mathrm{N} \mathrm{m}^{2} \times 10^{10}[28]$ & 9.8 \\
$S_{m} / \mathrm{J} \mathrm{K}{ }^{-1} \mathrm{Mol}^{-1}[21]$ & 30 \\
$h / \mathrm{nm} \mathrm{[21]}$ & 0.3368 \\
$\rho(\infty) / \mathrm{Kg} \mathrm{m}^{3} \times 10^{3}[29]$ & 2.329 \\
$a(\infty) / \mathrm{nm} \mathrm{[29]}^{-1}[29]$ & 5.431 \\
$v_{g}(\infty) / \mathrm{m} \mathrm{s}^{-1}[2]$ & 6524 \\
$\theta(\infty) / \mathrm{K}[21]$ & 692 \\
\hline
\end{tabular}

IV elements.

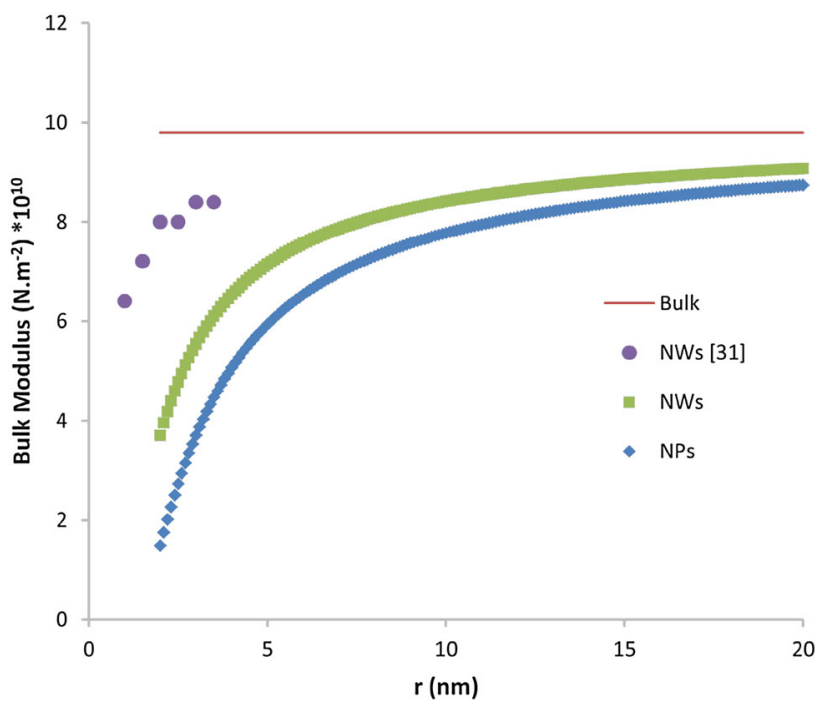

Figure 2. Bulk modulus for Si NPs and NWs dependence on the size compared with the results of MD simulation.

$$
\rho(r)=\rho(\infty) \frac{V(\infty)}{V(r)}
$$

where $\rho(\infty)$ is the bulk mass density and $\rho(r)$ the nanoscale size dependent mass density. 


\section{Analysis of results}

In this study, the necessary parameters for calculating bulk modulus of $\mathrm{Si}$ used are listed in table 1 . To confirm the feasibility of this method, calculated results are compared with the corresponding computer simulation results of $\mathrm{Si}$ NWs as well as the other related experimental data in nanoscale; such as melting point. The nanoscale size dependence for $B(r)$ is shown in figure 2 in terms of Eqs. (2), (3) and (8) for both Si NPs and NWs. Bulk modulus strongly depends on size which drops as the size of NCs decreases. The values of $B(r)$ are compared with the data calculated from young modulus $Y(r)$ obtained from molecular dynamic simulations (MDs) [30], that according to the relation $\mathrm{B}(\mathrm{r})=Y(r) / 3\left(1-2 P_{o}\right)$ [31], where $P_{o}$ is the bulk poissions ratio and is equal to 0.33 for $\mathrm{Si}$. The dependence exhibits the same trend variation to Si NWs but at lower values, as shown in figure 2 . In the nanosize range and according to the results obtained from $V(r)$ in this work, the unit cell volume are sensitively larger than that of bulk state. The higher values expected due to the assumption of constant lattice parameter in the calculation of MDs. In contrast, the variation of bulk modulus which is reported by Cherian et al [32] for semiconductor NCs, predicted the increases of bulk modulus with the reduction of materials size using Quantum Monte Carlo calculations within local density approximation. For group IV elemental semiconductors, the melting point $T_{m}(r)$ will follow a systematic dependence with their $\mathrm{B}(\mathrm{r})$ as shown in figure 3 . According to this phenomenon, the smaller size for NPs which relates to a lower melting temperature, $T_{m}(r)$ should consequently gives a smaller bulk modulus values $B(r)$ as it is the case in figure 4 using Eqs. (2) and (5).

From the physical point of view, the calculated size dependence bulk modulus $B(r)$ for NCs from the parameters $\rho(r)$ and $a(r)$ can be explained as follows; $\rho(r)$ decreases with the reduction of size for both Si NPs and NWs, which leads to a decrease in bulk modulus as shown in figure 5 in terms of Eq. (8) for Si NPs. For a $5 \mathrm{~nm}$ size, the density value of $\mathrm{Si}$ NPs is found to be $1.91 \times 10^{3} \mathrm{~kg} \mathrm{~m}^{3}$ compared to $2.329 \times 10^{3} \mathrm{~kg} \mathrm{~m}^{3}$ for bulk; this is largely due to the variation in the surface to volume ratio which leads to increase in mean bond length according to Eqs. (6) and (7). On the other hand, with the reduction of size, more atoms will be located on the surface and thus, $\mathrm{A} / \mathrm{V}$ increases with decreasing $\mathrm{r}$ which is related to a volume change. It is well known that for solids the lattice volume has a major effect on physical properties such as electronic band structure, electrical, thermal and elastic properties. That is why the actual lattice volume is of great importance for the use of nanoscale range materials in technology.

Furthermore, for bulk crystals, lattice parameter is constant; it means that the mean bond length is also constant. Thus, the volume is constant but any change in lattice volume leads to a change in properties of the materials. The

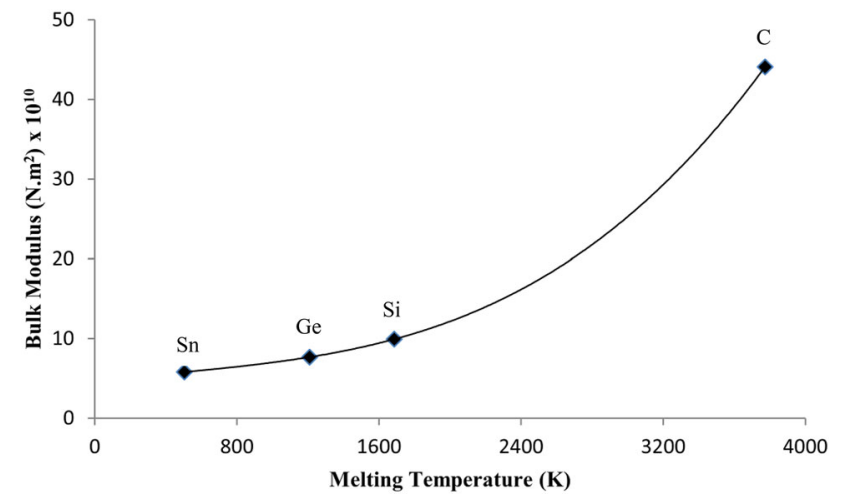

Figure 3. Melting point versus on bulk modulus for different group.

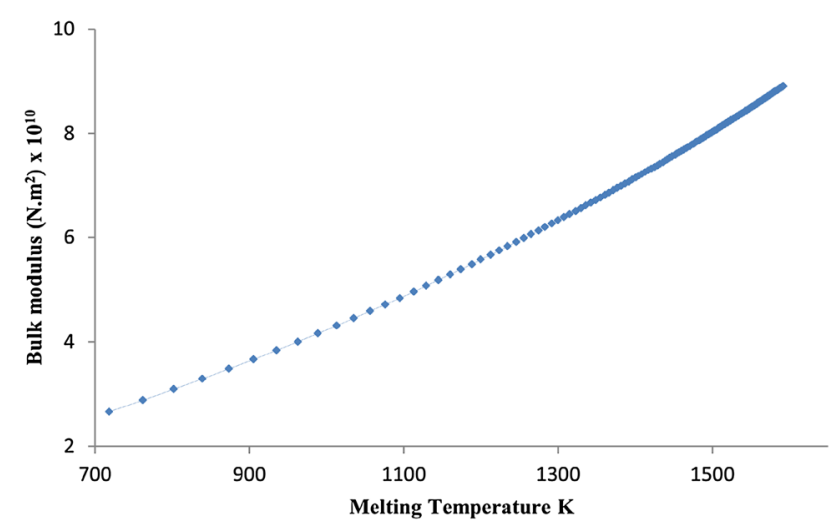

Figure 4. Melting point versus bulk modulus for $\mathrm{Si}$ nanoparticles.

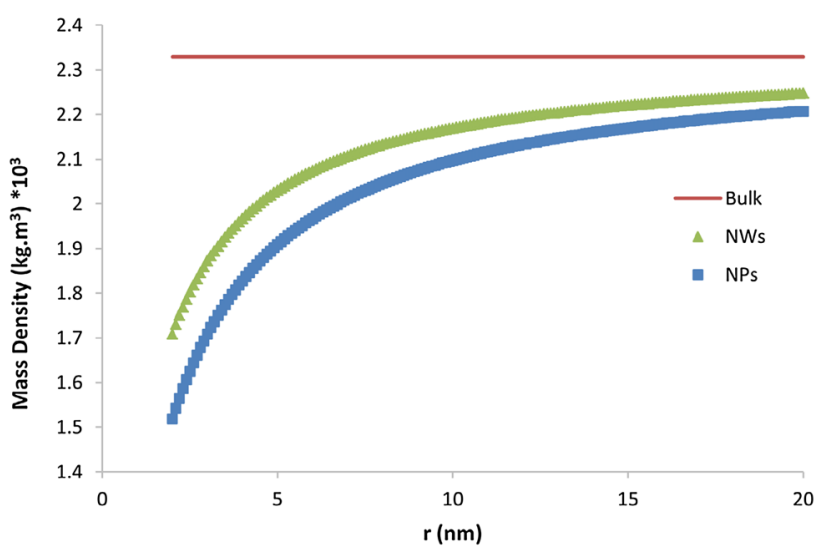

Figure 5. Size dependent mass density for Si NPs and NWs.

same trend variations of the expansion of lattice constant have been reported by [33]. Mass density and lattice volume have no effect on bulk and microstructure due to the existence of more core atoms compared to that of the surface, the effects appeared for a material size of several nanometers and less. For nanoscale range, the lattice 
parameter is size-dependent and it increases with the reduction of size, it leads to variation in volume ratio $V_{(D)} / V_{(\infty)}$. Density is a known size-independent property for both bulk and microstructure materials. In general, density can be altered by changing either the pressure or the temperature. Recent experimental studies have shown that mass density is a size-dependent property of nanomaterials, for example, Siegel et al [34] reported the decreases of mass density for $\mathrm{Au}$ NFs as its thickness reaches the nanometer scale. Opalinska et al [35] reported the decrease of mass density for monoclinic $\mathrm{ZrO}_{2}$ NPs with decreasing its size, particularly at a diameter of $<20 \mathrm{~nm}$. Several models have been established to calculate the dependence of lattice parameters on the size of nanomaterials. Omar [22] found an increase in lattice constant in nanomaterials through calculations for the mean bond length. Zhu et al [33] also obtained a similar result, which is due to the relative disordered arrangement of the atoms and the volume-induced stress field and overall lattice expansion. The same results were obtained by Shen [25] as theoretical prediction calculations on $\mathrm{Si}$ nanostructures. Conversely, crystal lattice in niobium $(\mathrm{Nb})$ nanostructures increases significantly with reduction of its crystalline size, particularly for size below $10 \mathrm{~nm}[36,37]$, at which the lattice expansion increases with reduction in the average grain or crystalline size.

\section{Conclusions}

The method to determine the size effect on bulk modulus at nanoscale region materials is based on the ratio number of surface atoms to that of its interior as applied on Si. The nanosize dependence in solids on mass density and melting temperature explains well the decrease in the bulk of a nanometic scale for both NWs and NPs. In the nanoscale range, melting point and mass density depend strongly on surface to volume ratio. The mass density decreases with decreasing size and the results indicated that the variation is largely due to the differences in variations of lattice parameter.

\section{Acknowledgement}

The financial support from Ministry of Higher Education and Scientific Research- Salahaddin-Erbil University with the cooperation of Jilin University are acknowledged.

\section{References}

[1] Sun C Q 2009 Thermo-mechanical behavior of low-dimensional systems: The local bond average approach. Prog. Mater. Sci. 54: 179-307
[2] Gleiter H 2000 Nanostructured materials: basic concepts and microstructure. Acta Materialia 48: 1-29

[3] Zhao M and Jiang Q 2010 Size effect on thermal properties in low-dimensional materials. Key Engineering Materials, Trans Tech Publications, 189-218

[4] Zhang Z, Zhao M and Jiang Q 2001 Melting temperatures of semiconductor nanocrystals in the mesoscopic size range. Semicond. Sci. Technol. 16:L33

[5] Qi W 2005 Size effect on melting temperature of nanosolids. Physica B: Condens. Matter. 368: 46-50

[6] Zhu Y, Lian J and Jiang Q 2009 Modeling of the melting point, Debye temperature, thermal expansion coefficient, and the specific heat of nanostructured materials. J. Phys. Chem. C 113: 16896-16900

[7] Lu H, Li P, Cao Z and Meng X 2009 Size-, shape-, and dimensionality-dependent melting temperatures of nanocrystals. J. Phys. Chem. C 113: 7598-7602

[8] Hou M, Elazzaoui M, Pattyn H, Verheyden J, Koops, G and Zhang G 2000 Growth and lattice dynamics of Co nanoparticles embedded in Ag: a combined molecular-dynamics simulation and Mössbauer study. Phys. Rev. B 62: 5117

[9] Yang C, Xiao M, Li W and Jiang Q 2006 Size effects on Debye temperature, Einstein temperature, and volume thermal expansion coefficient of nanocrystals. Solid State Communications 139: 148-152

[10] Abdullah B J, Omar M S and Jiang Q 2017 Size effects on cohesive energy, Debye temperature and lattice heat capacity from first-principles calculations of $\mathrm{Sn}$ nanoparticles. Proceedings of the National Academy of Sciences, India Section A: Physical Sciences, 1-4

[11] Kittel C 2005 Introduction to solid state physics. Wiley, Hoboken, 25

[12] Koc H, Mamedov A M, Deligoz E and Ozisik H 2012 First principles prediction of the elastic, electronic, and optical properties of Sb2S3 and $\mathrm{Sb} 2 \mathrm{Se} 3$ compounds. Solid State Sci. 14: $1211-1220$

[13] Gacem A, Doghmane A and Hadjoub Z 2011 Quantification the Effect of the Thickness of Thin Films on their Elastic Parameters. Adv. Mater. Res. Trans. Tech. Pub. 93-96

[14] Qiao Z, Latz R and Mergel D 2004 Thickness dependence of In2O3: Sn film growth. Thin Solid Films 466: 250-258

[15] Safaei A 2012 Size-dependent mass density of nanocrystals. Nano 7: 1250009

[16] Lovell S and Rollinson E 1968 Density of thin films of vacuum evaporated metals. Nature 218: 1179-1180

[17] Gu M, Sun C Q, Chen Z, Yeung T A, LI S, Tan C and Nosikn V 2007 Size, temperature, and bond nature dependence of elasticity and its derivatives on extensibility, Debye temperature, and heat capacity of nanostructures. Phys. Rev. B 75: 125403

[18] Zhao M and Jiang Q 2004 Melting and surface melting of low-dimensional in crystals. Solid State Commun. 130: 37-39

[19] Liang L and Li B 2006 Size-dependent thermal conductivity of nanoscale semiconducting systems. Phys. Rev. B 73: 153303

[20] Avramov I and Michailov M 2008 Specific heat of nanocrystals. J. Phys. Condens. Matter. 20: 295224

[21] Omar M 2012 Models for mean bonding length, melting point and lattice thermal expansion of nanoparticle materials. Mater. Res. Bull. 47: 3518-3522 
[22] Omar M 2007 Lattice thermal expansion for normal tetrahedral compound semiconductors. Mater. Res. Bull. 42: 319-326

[23] Jiang Q, Shi H and Zhao M 1999 Melting thermodynamics of organic nanocrystals. J. Chem. Phys. 111: 2176-2180

[24] Zhang Z, Li J and Jiang Q 2000 Modelling for size-dependent and dimension-dependent melting of nanocrystals. $J$. Phys. D Appl. Phys. 33: 2653

[25] Shen T D, Zhang J and Zhao Y 2008 What is the theoretical density of a nanocrystalline material? Acta Materialia 56: 3663-3671

[26] Abdullah B J, Jiang Q and Omar M S 2016 Effects of size on mass density and its influence on mechanical and thermal properties of $\mathrm{ZrO} 2$ nanoparticles in different structures. Bull. Mater. Sci. 39: 1295-1302

[27] Lam P K, Cohen M L and Martinez G 1987 Analytic relation between bulk moduli and lattice constants. Phys. Rev. B 35: 9190-9194

[28] Bievere P D, Valkiers S, Peiser S, Becker P, Ludicke F, Spieweck F and Stumpel J 1995 A more accurate value for the Avogadro constant. IEEE Trans. Instrum. Meas. 44: 530-532

[29] Goldstein A N 1996 The melting of silicon nanocrystals: Submicron thin-film structures derived from nanocrystal precursors. Appl. Phys. A 62: 33-37
[30] Kang K and Cai W 2010 Size and temperature effects on the fracture mechanisms of silicon nanowires: Molecular dynamics simulations. Int. J. Plast. 26: 1387-1401

[31] Wolf H F 1971 Semiconductors. Wiley, Hoboken, 128

[32] Cherian R, Gerard C, Mahadevan P, Cuong N T and Maezono R 2010 Size dependence of the bulk modulus of semiconductor nanocrystals from first-principles calculations. Phys. Rev. B 82: 235321

[33] Zhu Y, Zheng W and Jiang Q 2009 Modeling lattice expansion and cohesive energy of nanostructured materials. Appl. Phys. Lett. 95: 083110

[34] Siegel J, Lyutakov O, Rybka V, Kolská Z and Svorcík V 2011 Properties of gold nanostructures sputtered on glass. Nanoscale Res. Lett. 6: 96

[35] Opalinska A, Malka I, Dzwolak W, Chudoba T, Presz A and Lojkowski W 2015 Size-dependent density of zirconia nanoparticles. Beilstein J. Nanotechnol. 6: 27-35

[36] Banerjee R, Sperling E A, Thompson G B, Fraser H L, Bose S and Ayyub P 2003 Lattice expansion in nanocrystalline niobium thin films. Appl. Phys. Lett. 82: 4250-4252

[37] Chattopadhyay P P, Nambissan P M G, Pabi S K and Manna I 2001 Polymorphic bcc to fcc transformation of nanocrystalline niobium studied by positron annihilation. Phys. Rev. B 63: 054107 